\title{
SURFACES ISOGENOUS TO A PRODUCT OF CURVES, BRAID GROUPS AND MAPPING CLASS GROUPS
}

\author{
MATTEO PENEGINI
}

\section{Contents}

0. Introduction

1. Braid Group and Mapping Class Group

2. Surfaces Isogenous to a Product of Curves

3. Connected components of the Moduli Space of Surfaces of General Type 10 References

\section{INTRODUCTION}

This article is a revised version of the talk I gave at the conference "Beauville Surfaces and groups" held in Newcastle in June 2012. It presents some group theoretical methods to give bounds on the number of connected components of the moduli space of surfaces of general type, focusing on some families of regular surfaces isogenous to a product of curves. Some of the results appearing in this work have been proven in collaboration with Shelly Garion.

We will use the standard notation from the theory of complex algebraic surfaces. Let $S$ be a smooth, complex, projective, minimal surface $S$ of general type; this means that the canonical divisor $K_{S}$ of $S$ is big and nef.

The principal numerical invariants for the study of minimal surfaces of general type are

- the geometric genus $p_{g}(S):=h^{0}\left(S, \Omega_{S}^{2}\right)=h^{0}\left(S, \mathcal{O}_{S}\left(K_{S}\right)\right)$,

- the irregularity $q(S):=h^{0}\left(S, \Omega_{S}^{1}\right)$, and

- the self intersection of the canonical divisor $K_{S}^{2}$.

In fact, these determine all the other classical invariants, as

- the Euler-Poincaré characteristic $\chi\left(\mathcal{O}_{S}\right)=1-q(S)+p_{g}(S)$,

- the topological Euler number $e(S)=12 \chi(S)-K_{S}^{2}$, and

- the plurigenera $P_{n}(S)=\chi(S)+\left(\begin{array}{l}n \\ 2\end{array}\right) K_{S}^{2}$.

Moreover, we call a surface regular if its irregularity vanishes, i.e., $q(S)=0$.

By a theorem of Bombieri, a minimal surface of general type $S$ with fixed invariants is birationally mapped to a normal surface $X$ in a fixed projective space of dimension $P_{5}(S)-1$. Moreover, $X$ is uniquely determined and is called the canonical model of $S$. Let us recall Gieseker's Theorem.

Theorem 0.1. There exists a quasi-projective coarse moduli space $\mathcal{M}_{y, x}$ for canonical models of surfaces of general type $S$ with fixed invariants $y:=K_{S}^{2}$ and $x:=\chi$.

The union $\mathcal{M}$ over all admissible pairs of invariants $(y, x)$ of these spaces is called the moduli space of surfaces of general type. If $S$ is a smooth minimal surface of general type, we denote by $\mathcal{M}(S)$ the subvariety of $\mathcal{M}_{y, x}$, corresponding to surfaces (orientedly) homeomorphic to $S$. Moreover, we denote by $\mathcal{M}_{y, x}^{0}$ the subspace of the moduli space corresponding to regular surfaces.

It is known that the number of connected components $\delta(y, x)$ of $\mathcal{M}_{y, x}^{0}$ is bounded from above by a function of $y$; more precisely by Cat92] we have $\delta(y, x) \leq c y^{77 y^{2}}$, where $c$ is a positive 
constant. Hence we have that the number of components has an exponential upper bound in $K^{2}$.

There are also some results regarding the lower bound. In M97, for example, Manetti constructed a sequence $S_{n}$ of simply connected surfaces of general type, such that the lower bound for the number of the connected components $\delta\left(S_{n}\right)$ of $\mathcal{M}\left(S_{n}\right)$ is given by

$$
\delta\left(S_{n}\right) \geq y_{n}^{\frac{1}{5} \log y_{n}} .
$$

Using group theoretical methods we are able to describe the asymptotic growth of the number of connected components of the moduli space of surfaces of general type relative to certain sequences of surfaces. More precisely, we apply the definition and some properties of regular surfaces isogenous to a product of curves and of some special cases of them, Beauville surfaces, to reduce the geometric problem of finding connected components into the algebraic one of counting orbits of some group action, which can be effectively computed.

The paper is organized as follows.

In the first Section we recall the definition and some properties of the mapping class group. Moreover, we describe the Hurwitz moves in the most general setting.

In the second Section we briefly recall the definition of surfaces isogenous to a product of curves. We will recall some of the properties of these surfaces focusing on their moduli space.

In the third part we present some results obtained with Shelly Garion about the number of connected components of the moduli space of surfaces isogenous to a product.

Finally, we also point out some possible future developments.

Acknowledgments. The author is grateful to G. Bini for reading and commenting the paper. Moreover the author thanks the organizers of the conference Beauville surfaces and Groups N. Barker, I. Bauer, S. Garion and A. Vdovina for the invitation and the kind hospitality.

\section{Braid Group and Mapping Class Group}

In this section, we first recall the definition of mapping class group. Next we give a presentation of it for $\mathbb{P}^{1}-\left\{p_{1}, \ldots, p_{r}\right\}$, and more generally for a curve of genus $g^{\prime}$ with $r$ marked points. After that, we calculate the Hurwitz moves induced by those groups. We mainly follow the definitions and notation of [Cat03a].

Definition 1.1. Let $M$ be a differentiable manifold, then the mapping class group of $M$ is the group:

$$
\operatorname{Map}(M):=\pi_{0}\left(\operatorname{Diff}^{+}(M)\right):=\operatorname{Diff}^{+}(M) / \operatorname{Diff}^{0}(M),
$$

where $\operatorname{Diff}^{+}(M)$ is the group of orientation preserving diffeomorphisms of $M$ and $\operatorname{Diff}^{0}(M)$ is the subgroup of diffeomorphisms of $M$ isotopic to the identity.

If $M$ is a compact complex curve of genus $g^{\prime}$ we use the following notation:

(1) We denote the mapping class group of $M$ without marked points by $\mathrm{Map}_{g^{\prime}}$.

(2) If we consider $r$ unordered marked points $p_{1}, \ldots, p_{r}$ on $M$ we define:

$$
\operatorname{Map}_{g^{\prime},[r]}=\pi_{0}\left(\operatorname{Diff}^{+}\left(M-\left\{p_{1}, \ldots, p_{r}\right\}\right)\right),
$$

and this is known as the full mapping class group.

There is a way to present the full mapping class group of a curve using three different types of twists.

Theorem 1.2. The mapping class group $\operatorname{Map}_{0,[r]}=\pi_{0}\left(\operatorname{Diff}^{+}\left(\mathbb{P}^{1}-\left\{p_{1}, \ldots, p_{r}\right\}\right)\right)$ is isomorphic to the braid group $\mathbf{B}_{r}$ on $r$ strands, which can be presented as

$$
\left.\mathbf{B}_{r}=\left\langle\sigma_{1}, \ldots, \sigma_{r-1}\right| \sigma_{i} \sigma_{i+1} \sigma_{i}=\sigma_{i+1} \sigma_{i} \sigma_{i+1}, \sigma_{i} \sigma_{j}=\sigma_{j} \sigma_{i} \text { if }|i-j| \geq 2\right\rangle .
$$

For a proof of the above Theorem see, for example, [Bir74, Theorem 1.11].

In this way Artin's standard generators $\sigma_{i}(i=1, \ldots, r-1)$ of $\mathbf{B}_{r}$ can be represented by the so-called half-twists. 
Definition 1.3. The half-twist $\sigma_{j}$ is a diffeomorphism of $\mathbb{P}^{1}-\left\{p_{1}, \ldots, p_{r}\right\}$ isotopic to the homeomorphism given by (see Figure 1):

- A rotation of 180 degrees on the disk with center $j+\frac{1}{2}$ and radius $\frac{1}{2}$;

- on a circle with the same center and radius $\frac{2+t}{4}$ the map $\sigma_{j}$ is the identity if $t \geq 1$ and a rotation of $180(1-t)$ degrees, if $t \leq 1$.
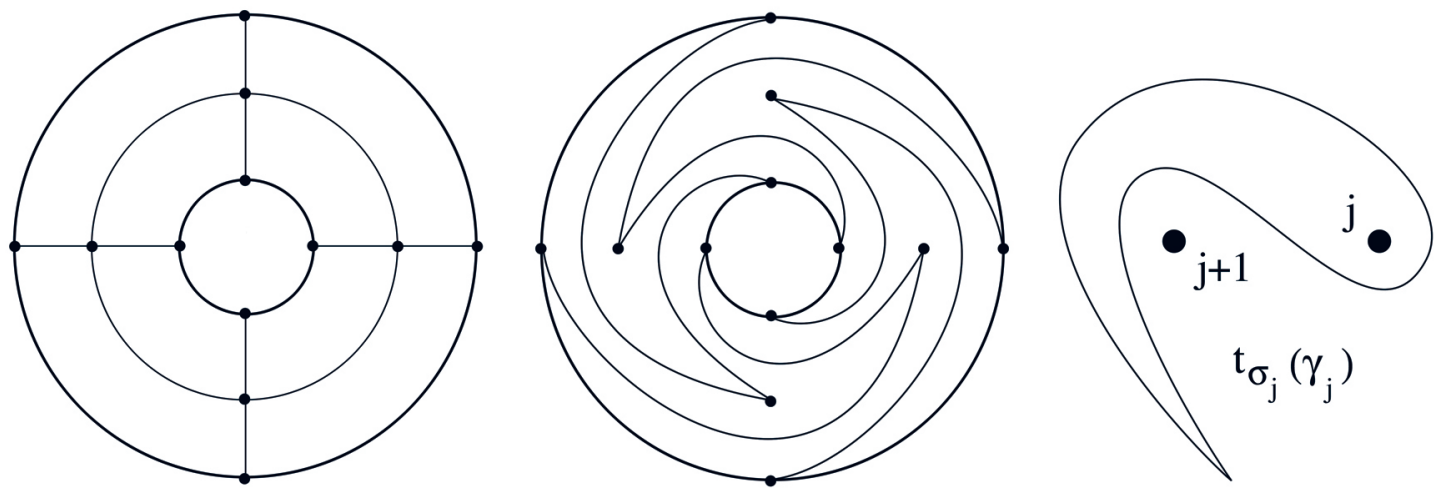

Figure 1.

We want to give a similar presentation for a group $\operatorname{Map}_{g^{\prime}}$ with $g^{\prime} \geq 1$, so we have to introduce the Dehn twists.

Definition 1.4. Let $C$ be an oriented Riemann surface. Then a positive Dehn twist $t_{\alpha}$ with respect to a simple closed curve $\alpha$ on $C$ is an isotopy class of a diffeomorphism $h$ of $C$ which is equal to the identity outside a neighborhood of $\alpha$ orientedly homeomorphic to an annulus in the plane, while inside the annulus $h$ rotates the inner boundary of the annulus by $360^{\circ}$ to the right and damps the rotation down to the identity at the outer boundary (see Figure 2).
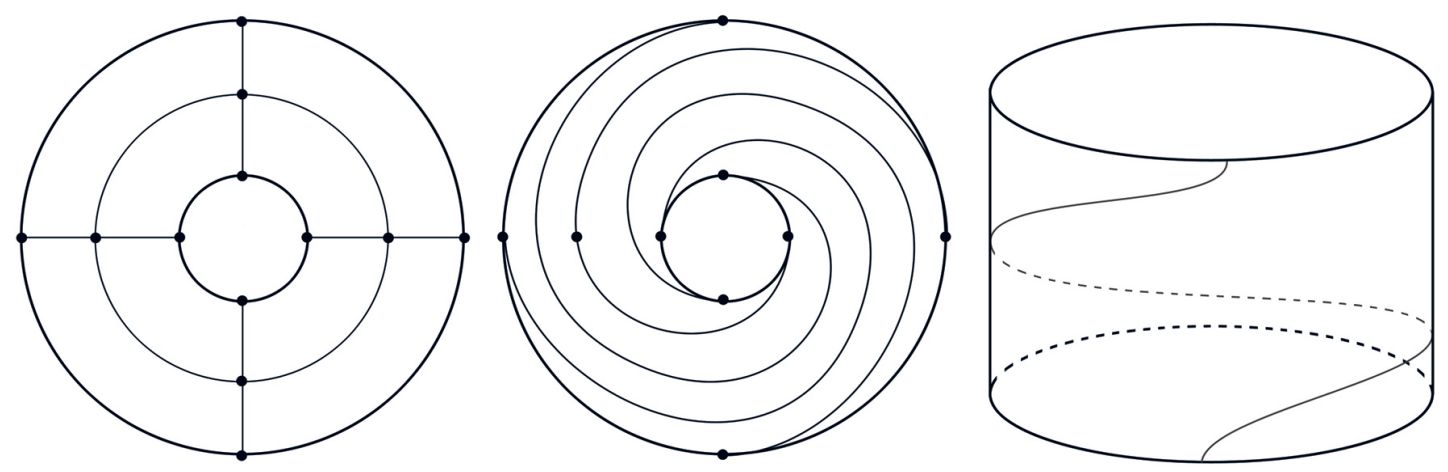

Figure 2.

We have then the following classical results of Dehn [D38].

Theorem 1.5. The mapping class group $\mathrm{Map}_{g^{\prime}}$ is generated by Dehn twists.

We give the generators of the group $\mathrm{Map}_{g^{\prime}}$.

Theorem 1.6. The group $\mathrm{Map}_{g^{\prime}}$ is generated by the Dehn twists with respect to the curves in the Figure 3. 


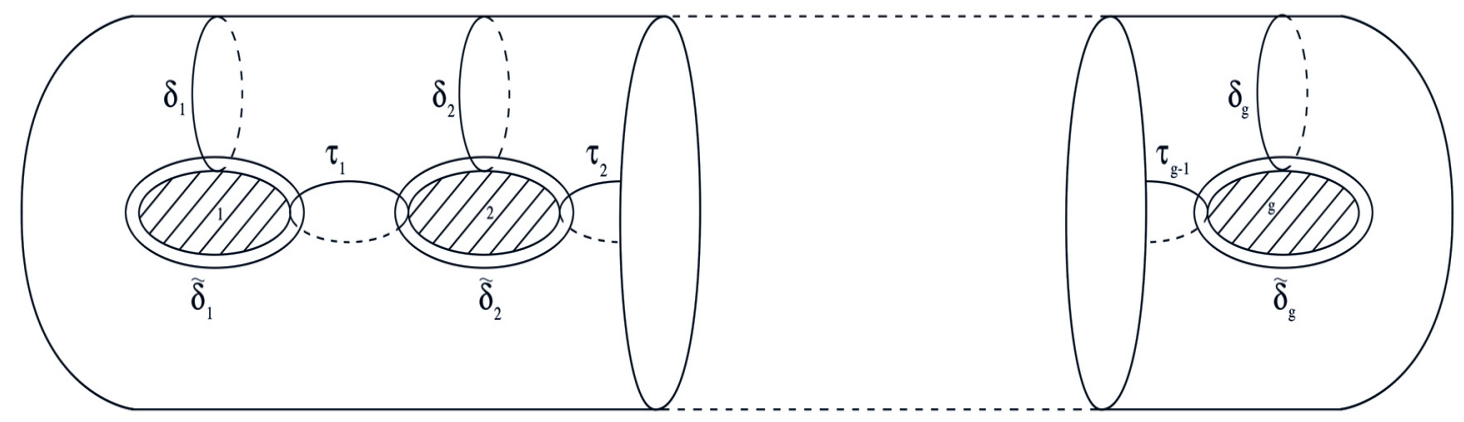

Figure 3.

A proof of the above Theorem can be found in [Bir74, Theorem 4.8].

For a more general situation where the surface has $g^{\prime}>0$ and $r$ marked points we need to introduce a third type of twist: The $\xi$-twists, which link the holes with the marked points. Let us recall the Birman short exact sequence for a Riemann surface $C^{\prime}$ with $g\left(C^{\prime}\right)>1$ or $r>1$, setting $\mathcal{B}:=\left\{p_{1}, \ldots, p_{r}\right\}$ :

$$
1 \longrightarrow \pi_{1}\left(C^{\prime} \backslash \mathcal{B}, p\right) \stackrel{\Xi}{\longrightarrow} \pi_{0}\left(\operatorname { D i f f } ^ { + } ( C ^ { \prime } \backslash ( \mathcal { B } \cup \{ p \} ) ) \longrightarrow \pi _ { 0 } \left(\operatorname{Diff}^{+}\left(C^{\prime} \backslash \mathcal{B}\right) \longrightarrow 1 .\right.\right.
$$

The map $\Xi$ can be described as follows (cf. [Bir69]). Let $[\gamma] \in \pi_{1}\left(C^{\prime} \backslash \mathcal{B}, p\right)$ and $\gamma$ be a simple, smooth loop based at $p$ representing $[\gamma]$. Then $\Xi([\gamma])$ is the isotopy class of a $\xi$-twist with respect to the closed curve $\gamma$. In addition, this new twist is isotopic to the identity in $\pi_{0}\left(\operatorname{Diff}^{+}(C \backslash(\mathcal{B}))\right.$. Let us now describe a $\xi$-twist. We shall consider the annulus $A:=\left\{z=\rho e^{i \theta} \in \mathbb{C} \mid 1 \leq \rho \leq 2\right\}$, and we define $h: A \rightarrow A$ as follows

$$
h(\rho, \theta): \begin{cases}(\rho, \theta-4 \pi(\rho-1)) & 1 \leq \rho \leq \frac{3}{2} \\ (\rho, \theta-4 \pi(2-\rho)) & \frac{3}{2} \leq \rho \leq 2 .\end{cases}
$$

Definition 1.7. Let $C$ be a Riemann surface, and $\alpha$ a simple closed curve on $C$. Let $\iota$ be a diffeomorphism between $A$ and a tubular neighborhood of $\alpha$. Then the $\xi$-twist $\mathbf{t}_{\alpha}$ with respect to $\alpha$ is defined as $\left.\iota \circ h \circ \iota^{-1}\right|_{\iota(A)}$ extended to the whole $C$ as the identity on $C \backslash \iota(A)$ (see Figure 4).
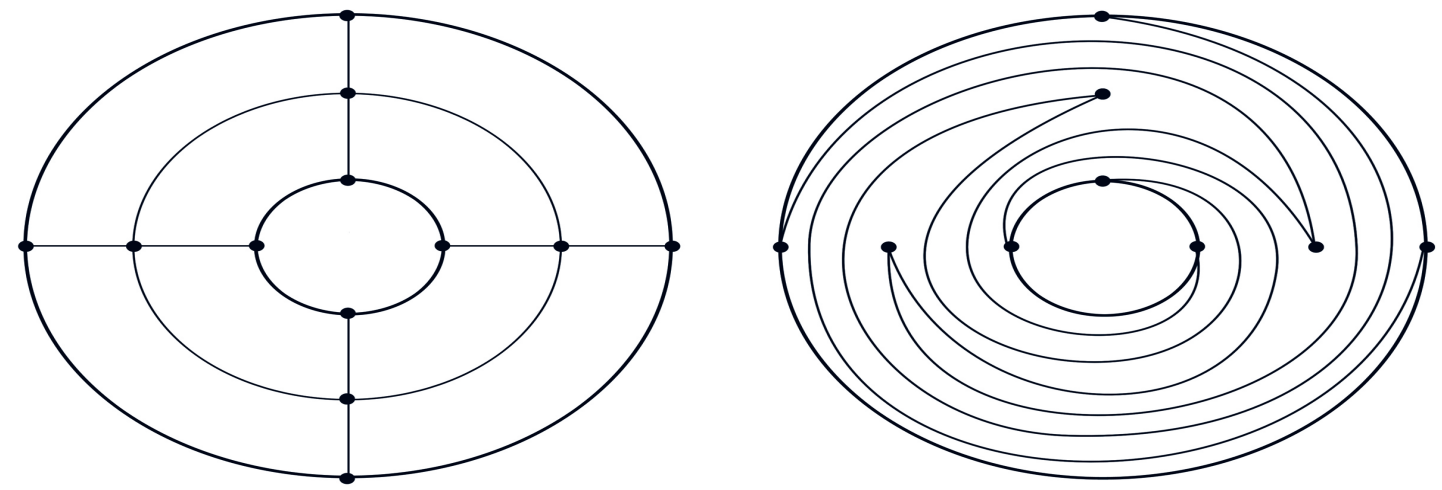

Figure 4.

Therefore, for the more general situation we have the following.

Theorem 1.8. [Bir69, Theorem 3] Let $g\left(C^{\prime}\right) \neq 0$ and $g\left(C^{\prime}\right)>1$ or $r>1$ then the group $\operatorname{Map}_{g^{\prime},[r]}$ is generated by the $3 g^{\prime}-1$ Dehn twists with respect to the curves $\delta_{j}, \tilde{\delta}_{j}$ and $\tau_{j}$, by the $2 r g^{\prime} \xi$-twists with respect to the curves $\xi_{j, d}^{l}$ and the $r-1$ half-twists about the points $p_{1}, \ldots, p_{r}$ in Figure 5. 


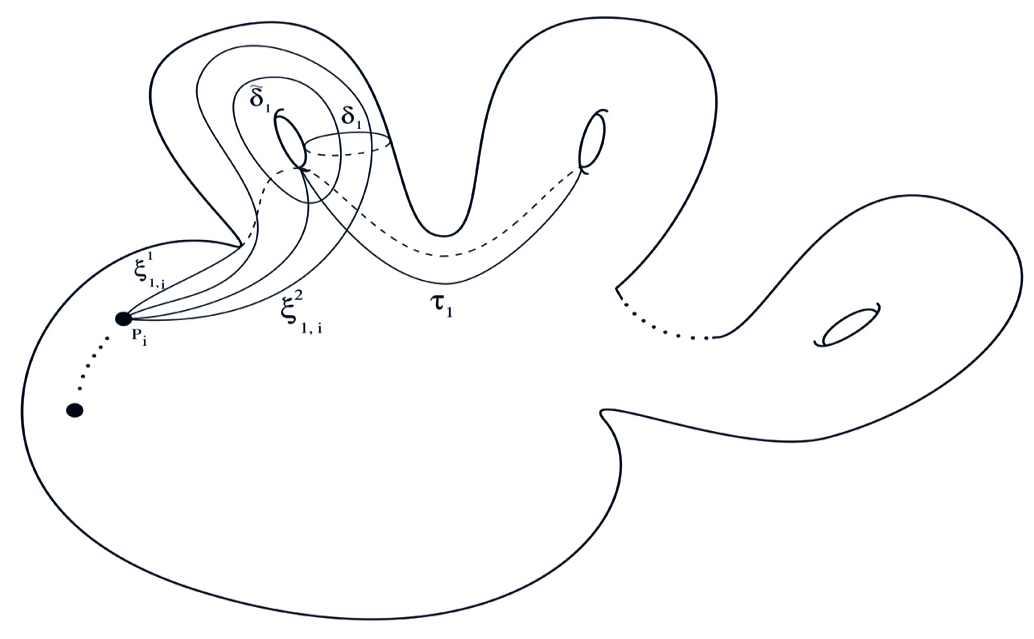

Figure 5.

Let $C^{\prime}$ be a Riemann surfaces of genus $g^{\prime}$, and let $\mathcal{B}:=\left\{p_{1}, \ldots, p_{r}\right\}$ a set of points on $C^{\prime}$. A geometric basis of $\pi_{1}\left(C^{\prime} \backslash \mathcal{B}, p_{0}\right)$ consists of simple non-intersecting (away from the base point) loops (see Figure 6)

$$
\gamma_{1}, \ldots, \gamma_{d}, \alpha_{1}, \beta_{1}, \ldots, \alpha_{g^{\prime}}, \beta_{g^{\prime}}
$$

such that we get the presentation

$$
\pi_{1}\left(C \backslash \mathcal{B}, p_{0}\right):=\left\langle\alpha_{1}, \beta_{1}, \ldots, \alpha_{g^{\prime}}, \beta_{g^{\prime}}, \gamma_{1}, \ldots, \gamma_{r} \mid \gamma_{1} \cdot \ldots \cdot \gamma_{r} \cdot \prod_{k=1}^{g^{\prime}}\left[\alpha_{k}, \beta_{k}\right]=1\right\rangle .
$$

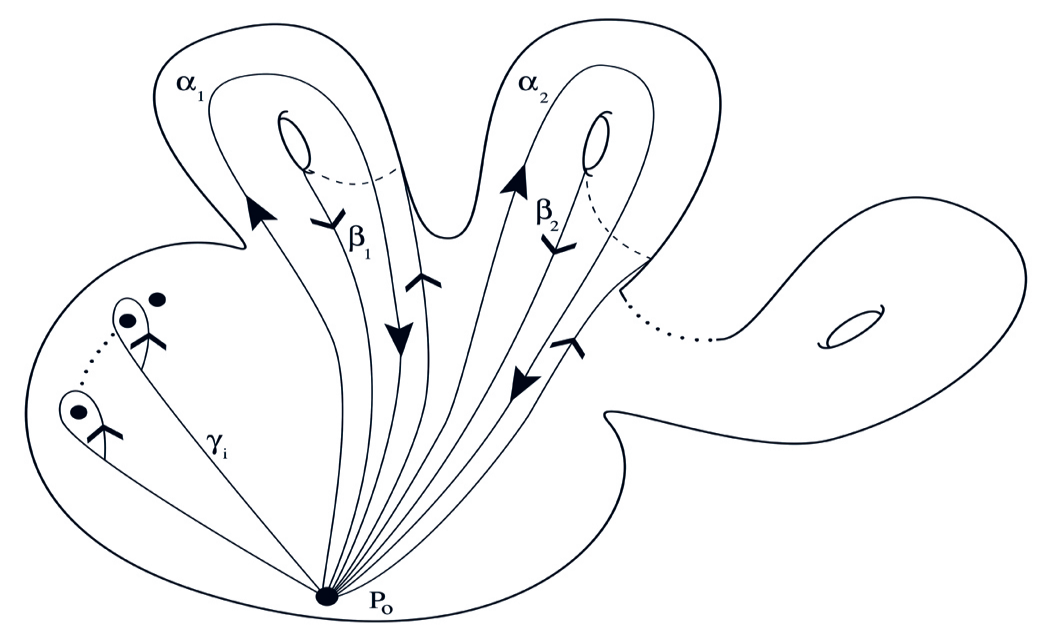

Figure 6.

Definition 1.9. Let $g^{\prime}, m_{1}, \ldots, m_{r}$ be positive integers. An orbifold surface group of type $\left(g^{\prime} \mid m_{1}, \ldots, m_{r}\right)$ is a group presented as follows:

$$
\begin{aligned}
\Gamma\left(g^{\prime} \mid m_{1}, \ldots, m_{r}\right):=\left\langle a_{1}, b_{1}, \ldots, a_{g^{\prime}}, b_{g^{\prime}}, c_{1}, \ldots, c_{r}\right| \\
\left.c_{1}^{m_{1}}=\cdots=c_{r}^{m_{r}}=c_{1} \cdot \ldots \cdot c_{r} \cdot \prod_{k=1}^{g^{\prime}}\left[a_{k}, b_{k}\right]=1\right\rangle .
\end{aligned}
$$

We notice that the choice of a geometric basis yields an obvious epimorphism $\pi_{1}\left(C \backslash \mathcal{B}, p_{0}\right) \rightarrow \Gamma\left(g^{\prime} \mid m_{1}, \ldots, m_{r}\right)$. 
The following is a reformulation of Riemann's existence theorem:

Theorem 1.10. A finite group $G$ acts as a group of automorphisms on some compact Riemann surface $C$ of genus $g$ if and only if there are natural numbers $g^{\prime}, m_{1}, \ldots, m_{r}$, and an orbifold homomorphism

$$
\theta: \Gamma\left(g^{\prime} \mid m_{1}, \ldots, m_{r}\right) \rightarrow G
$$

such that $\operatorname{ord}\left(\theta\left(c_{i}\right)\right)=m_{i}$ for all $i$ and such that the Riemann - Hurwitz relation holds:

$$
2 g-2=|G|\left(2 g^{\prime}-2+\sum_{i=1}^{r}\left(1-\frac{1}{m_{i}}\right)\right) .
$$

If this is the case, then $g^{\prime}$ is the genus of $C^{\prime}:=C / G$. The $G$-cover $C \rightarrow C^{\prime}$ is branched at $r$ points $p_{1}, \ldots, p_{r}$ with branching indices $m_{1}, \ldots, m_{r}$, respectively. Let $\Gamma=\Gamma\left(g^{\prime} \mid m_{1}, \ldots, m_{r}\right)$ be an orbifold surface group with a presentation as in Definition 1.9. If $G$ is a finite group quotient of $\Gamma$ as in (3), then we say that $G$ is $\left(g^{\prime} \mid m_{1}, \ldots, m_{r}\right)$-generated, the image of the generators of $\Gamma$ in $G$ is called a system of generators for $G$. Finally, $\theta$ is called an admissible epimorphism.

Definition 1.11. An automorphism $\eta \in \operatorname{Aut}(\Gamma)$ is said to be orientation preserving if the action induced on $\left\langle\alpha_{1}, \beta_{1}, \cdots, \alpha_{g^{\prime}}, \beta_{g^{\prime}}>^{a b}\right.$ has determinant +1 , and for all $i \in\{1, \ldots, r\}$ there exists $j$ such that $\eta\left(\gamma_{i}\right)$ is conjugate to $\gamma_{j}$, which implies ord $\left(\gamma_{i}\right)=\operatorname{ord}\left(\gamma_{j}\right)$.

The subgroup of orientation preserving automorphisms of $\Gamma$ is denoted by $\operatorname{Aut}^{+}(\Gamma)$ and the quotient $\mathrm{Out}^{+}(\Gamma):=\operatorname{Aut}^{+}(\Gamma) / \operatorname{Inn}(\Gamma)$ is called the mapping class group of $\Gamma$.

Theorem 1.12. Let $\Gamma=\Gamma\left(g^{\prime} \mid m_{1}, \ldots, m_{r}\right)$ be an orbifold surface group. Then there is an isomorphism of groups:

$$
\operatorname{Out}^{+}(\Gamma) \cong \operatorname{Map}_{g^{\prime},[r]} .
$$

This is a classical result cf. e.g., Macl74, §4] .

Moreover let $G$ be a finite group $\left(g^{\prime} \mid m_{1}, \ldots, m_{r}\right)$-generated. There is a section $s$ : $\mathrm{Out}^{+}(\Gamma) \rightarrow \operatorname{Aut}^{+}(\Gamma)$, which induces an action of the $\operatorname{Map}_{g^{\prime},[r]}$ on the generators of $\Gamma$. Such action does not depend on $s$ up to simultaneous conjugation, meaning that the action is defined up to inner automorphisms. This action induces an action on the systems of generators of $G$ via composition with admissible epimorphisms.

Definition 1.13. Let $G$ be a finite group $\left(g^{\prime} \mid m_{1}, \ldots, m_{r}\right)$-generated. If two systems of generators $\mathcal{V}_{1}$ and $\mathcal{V}_{2}$ are in the same $\mathrm{Map}_{g^{\prime},[r]}$-orbit, we say that they are related by a Hurwitz move (or are Hurwitz equivalent).

Proposition 1.14. Let $C^{\prime}$ be a curve of genus $g^{\prime}, \mathcal{B}:=\left\{p_{1}, \ldots, p_{r}\right\}$, and with $g^{\prime} \neq 0$ and $g^{\prime}>1$ or $r>1$. Up to inner automorphisms, the action of $\operatorname{Map}_{g^{\prime},[r]}$ on $\Gamma\left(g^{\prime} \mid m_{1} \ldots m_{r}\right)$ is induced by the following action on a geometric basis of $\pi_{1}\left(C^{\prime} \backslash \mathcal{B}, p_{0}\right)$

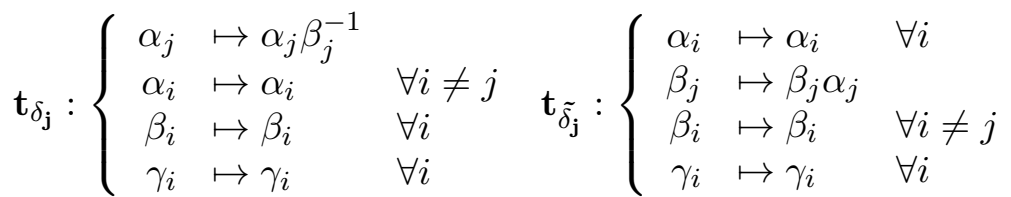

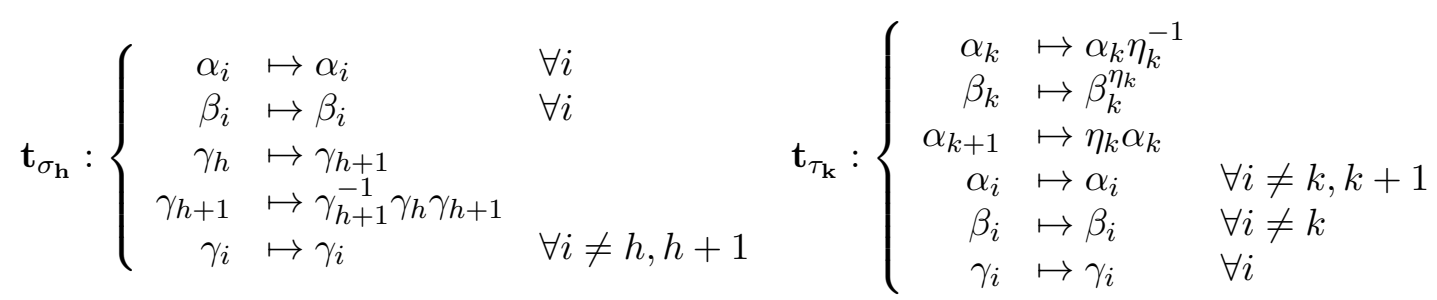




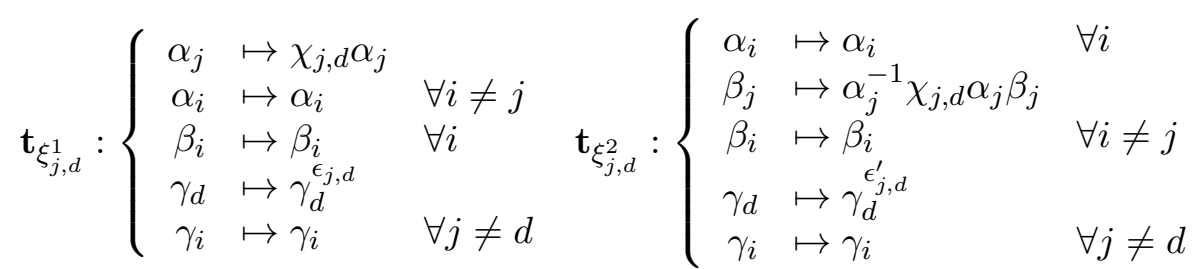

for $1 \leq j \leq g^{\prime}, 1 \leq k \leq\left(g^{\prime}-1\right), 1 \leq h \leq(r-1)$, and $1 \leq d \leq r$. Moreover we set $\eta_{k}:=\beta_{k}^{-1} \alpha_{k+1} \beta_{k+1} \alpha_{k+1}^{-1}, \chi_{j, d}:=\left(\Pi_{k=1}^{j-1}\left[\alpha_{k}, \beta_{k}\right]\right)^{-1} \gamma_{d} \Pi_{k=1}^{j-1}\left[\alpha_{k}, \beta_{k}\right]$, $\epsilon_{j, d}:=\gamma_{d}\left(\Pi_{k=1}^{j}\left[\alpha_{k}, \beta_{k}\right]\right) \alpha_{j} \beta_{j} \alpha_{j}^{-1}\left(\Pi_{k=1}^{j}\left[\alpha_{k}, \beta_{k}\right]\right)^{-1}$, and $\epsilon_{j, d}^{\prime}:=\gamma_{d}\left(\Pi_{k=1}^{j}\left[\alpha_{k}, \beta_{k}\right]\right) \alpha_{j}^{-1}\left(\Pi_{k=1}^{j}\left[\alpha_{k}, \beta_{k}\right]\right)^{-1}$.

In the above proposition the twists $\mathbf{t}_{\tilde{\delta}_{j}}, \mathbf{t}_{\delta_{j}}$ and $\mathbf{t}_{\tau_{j}}$ correspond to Dehn twists, $\mathbf{t}_{\xi_{r_{j, d}}}$ and $\mathbf{t}_{\xi_{r_{j, d}}}$ to $\xi$-twists, and finally $\mathbf{t}_{\sigma_{h}}$ to half-twists.

Proof. One notices that a Riemann surface of genus $g^{\prime}$ is a connected sum of $g^{\prime}$ tori. Then one can use the Figure 7 to calculate the Dehn twists about the curves $\delta_{j}$, and similarly for the Dehn twists about the curves $\tilde{\delta_{j}}$. One can use the results given in [P11] to calculate the Dehn twists about the curves $\tau_{j}$. In the Appendix of [CLP11 are described the actions of the $\xi$-twists. Finally the half-twists action is clear by Figure 1.

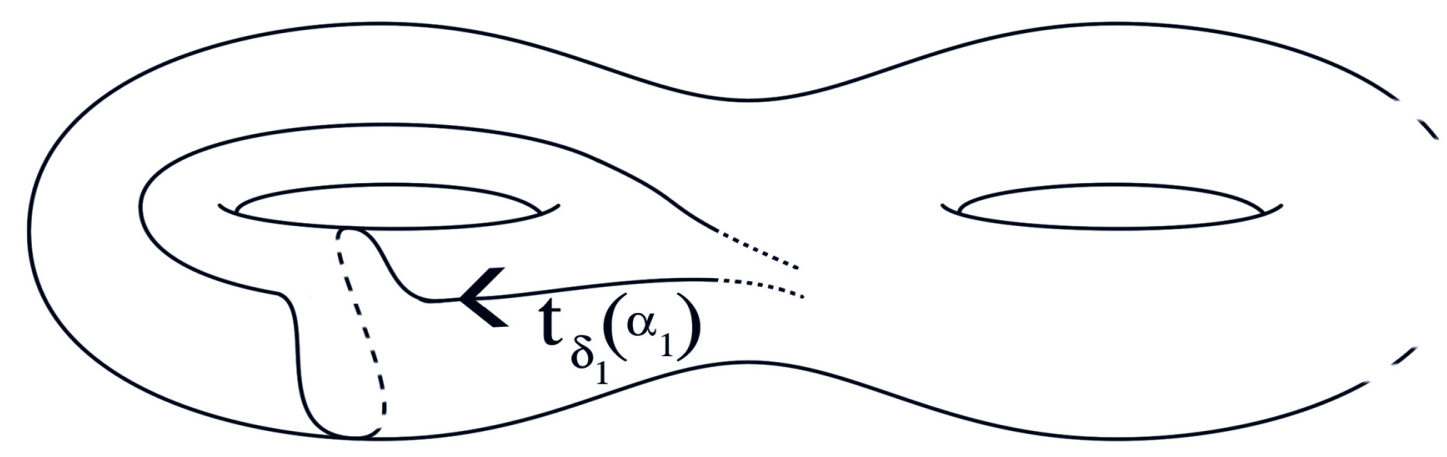

Figure 7.

For the case $g\left(C^{\prime}\right)=1$ and $r=1$ see e.g., [Po0] for a proof of the following proposition.

Proposition 1.15. [Po08, Proposition 1.10] Up to inner automorphisms, the action of $\mathrm{Map}_{1,1}$ on $\Gamma\left(1 \mid m_{1}\right)$ is induced by the following action on a geometric basis of $\pi_{1}\left(C^{\prime} \backslash \mathcal{B}, p_{0}\right)$

$$
\mathbf{t}_{\tilde{\delta}}:\left\{\begin{array}{ll}
\alpha_{1} & \rightarrow \alpha_{1} \\
\beta_{1} & \rightarrow \beta_{1} \alpha_{1} \\
\gamma_{1} & \rightarrow \gamma_{1}
\end{array} \quad \mathbf{t}_{\delta}:\left\{\begin{array}{l}
\alpha_{1} \rightarrow \alpha_{1} \beta_{1}^{-1} \\
\beta_{1} \rightarrow \beta_{1} \\
\gamma_{1} \rightarrow \gamma_{1} .
\end{array}\right.\right.
$$

Finally we have.

Proposition 1.16. Up to inner automorphism, the action of $\mathrm{Map}_{0,[r]}$ on $\Gamma\left(0 \mid m_{1}, \ldots, m_{r}\right)$ is induced by the following action on a geometric basis of $\pi_{1}\left(\mathbb{P}^{1} \backslash \mathcal{B}, p_{0}\right)$

for $i=1, \ldots, r-1$.

$$
\sigma_{i}:\left\{\begin{aligned}
\gamma_{i} & \rightarrow \gamma_{i+1} \\
\gamma_{i+1} & \rightarrow \gamma_{i+1}^{-1} \gamma_{i} \gamma_{i+1} \\
\gamma_{j} & \rightarrow \gamma_{j} \text { if } j \neq i, i+1
\end{aligned}\right.
$$

\section{Surfaces Isogenous to a Product of Curves}

A surface $S$ is said to be isogenous to a (higher) product of curves if and only if $S$ is a quotient $S:=\left(C_{1} \times C_{2}\right) / G$, where $C_{1}$ and $C_{2}$ are curves of genus at least two, and $G$ is a finite group acting freely on $C_{1} \times C_{2}$.

Let $S$ be a surface isogenous to a higher product, and $G^{\circ}:=G \cap\left(\operatorname{Aut}\left(C_{1}\right) \times \operatorname{Aut}\left(C_{2}\right)\right)$. Then $G^{\circ}$ acts on the two factors $C_{1}$ and $C_{2}$ and diagonally on the product $C_{1} \times C_{2}$. If $G^{\circ}$ 
acts faithfully on both curves, we say that $S=\left(C_{1} \times C_{2}\right) / G$ is a minimal realization of $S$. In Cat00, the author proves that any surface isogenous to a higher product admits a unique minimal realization. From now on, we work only with minimal realizations.

There are two cases: the mixed case where the action of $G$ exchanges the two factors (in this case $C_{1}$ and $C_{2}$ are isomorphic and $G^{\circ} \neq G$ ); the unmixed case (where $G=G^{\circ}$, and therefore it acts diagonally).

Moreover, we observe that a surface isogenous to a product of curves is of general type. It is always minimal and its numerical invariants are explicitly given in terms of the genera of the curves and the order of the group. Indeed, we have the following proposition.

Proposition 2.1. Let $S=\left(C_{1} \times C_{2}\right) / G$ be a surface isogenous to a higher product of curves, then:

$$
\chi(S)=\frac{\left(g\left(C_{1}\right)-1\right)\left(g\left(C_{2}\right)-1\right)}{|G|}, \quad e(S)=4 \chi(S), \quad K_{S}^{2}=8 \chi(S) .
$$

The irregularity of these surfaces is easily computed by

$$
q(S)=g\left(C_{1} / G\right)+g\left(C_{2} / G\right) .
$$

By the above formula a surface $S$ isogenous to a product of curves has $q(S)=0$ if and only if the two quotients $C_{i} / G$ are isomorphic to $\mathbb{P}^{1}$. Moreover, if both coverings $C_{i} \rightarrow C_{i} / G \cong \mathbb{P}^{1}$ are ramified in exactly 3 points, $S$ is a Beauville surface. This last condition is equivalent to saying that Beauville surfaces are rigid, i.e., have no nontrivial deformations.

In the unmixed case $G$ acts separately on $C_{1}$ and $C_{2}$, and the two projections $\pi_{i}: C_{1} \times C_{2} \longrightarrow$ $C_{i}$ for $i=1,2$ induce two isotrivial fibrations $\alpha_{i}: S \longrightarrow C_{i} / G$ for $i=1,2$, whose smooth fibres are isomorphic to $C_{2}$ and $C_{1}$, respectively. We work only with surfaces of unmixed type.

Working out the definition of surfaces isogenous to a product, one sees that there is a pure group theoretical condition which characterizes the groups of such surfaces: the existence of a "ramification structure".

Definition 2.2. Let $G$ be a finite group and $\theta_{1}: \Gamma\left(g_{1}^{\prime} \mid m_{1,1}, \ldots, m_{1, r_{1}}\right) \rightarrow G$ an admissible epimorphism. Let $\mathcal{V}_{1}$ be the system of generators of $G$ induced by $\theta_{1}$, i.e., the elements of $G$ which are images of the generators of $\Gamma$. We say that $\mathcal{V}_{1}$ is of type $\tau_{1}:=\left(g_{1}^{\prime} \mid m_{1,1}, \ldots, m_{1, r_{1}}\right)$.

Moreover, let $\theta_{2}: \Gamma\left(g_{2}^{\prime} \mid m_{2,1}, \ldots, m_{2, r_{2}}\right) \rightarrow G$ be another admissible epimorphism and $\mathcal{V}_{2}$ be the system of generators of $G$ induced by $\theta_{2}$. Then $\mathcal{V}_{1}$ and $\mathcal{V}_{2}$ are said to be disjoint, if:

$$
\Sigma\left(\mathcal{V}_{1}\right) \bigcap \Sigma\left(\mathcal{V}_{2}\right)=\{1\}
$$

where

$$
\Sigma\left(\mathcal{V}_{i}\right):=\bigcup_{g \in G} \bigcup_{j=0}^{\infty} \bigcup_{k=1}^{r_{i}} g \cdot \theta_{i}\left(\gamma_{k}\right)^{j} \cdot g^{-1} .
$$

Definition 2.3. Let $\tau_{i}:=\left(g_{i}^{\prime} \mid m_{1, i}, \ldots, m_{r_{i}, i}\right)$ for $i=1,2$ be two types. An unmixed ramification structure of type $\left(\tau_{1}, \tau_{2}\right)$ for a finite group $G$, is a pair $\left(\mathcal{V}_{1}, \mathcal{V}_{2}\right)$ of disjoint systems of generators of $G$, whose types are $\tau_{i}$, and they satisfy:

$$
\mathbb{Z} \ni \frac{|G|\left(2 g_{i}^{\prime}-2+\sum_{l=1}^{r_{i}}\left(1-\frac{1}{m_{i, l}}\right)\right)}{2}+1 \geq 2,
$$

for $i=1,2$.

We shall denote by $\mathcal{U}\left(G ; \tau_{1}, \tau_{2}\right)$ the set of all pairs $\left(\mathcal{V}_{1}, \mathcal{V}_{2}\right)$ of disjoint systems of generators of unordered type $\left(\tau_{1}, \tau_{2}\right)$. Here unordered type $\tau$ means that there is a permutation $\sigma \in \mathfrak{S}_{r}$ such that: $\operatorname{ord}\left(c_{1}\right)=m_{\sigma(1)}, \ldots, \operatorname{ord}\left(c_{r}\right)=m_{\sigma(r)}$. We obtain that the datum of a surface isogenous to a higher product of unmixed type $S=\left(C_{1} \times C_{2}\right) / G$ is determined, looking at the monodromy of each covering of $C_{i} / G$, by the datum of a finite group $G$ together with an unmixed ramification structure. The condition (7) ensures that the action of $G$ on the product of the two curves $C_{1} \times C_{2}$ is free. We remark here that this can be specialized to $C_{i} / G \cong \mathbb{P}^{1}$ in order to obtain regular surfaces isogenous to a product. In this case condition (8) is automaticaly satisfied, 
see [GP11, Lemma 2.4]. Moreover, we can also ask $r_{i}=3$, and therefore we obtain Beauville surfaces, in this case the ramification structure of $G$ is called a Beauville ramification structure.

Remark 2.4. Note that a group $G$ and an unmixed ramification structure (or equivalently a Beauville structure) determine the main invariants of the surface $S$. Indeed, by (5) and (4) we obtain:

$$
4 \chi(S)=|G| \cdot\left(2 g_{1}^{\prime}-2+\sum_{k=1}^{r_{1}}\left(1-\frac{1}{m_{1, k}}\right)\right) \cdot\left(2 g_{2}^{\prime}-2+\sum_{k=1}^{r_{2}}\left(1-\frac{1}{m_{2, k}}\right)\right),
$$

and so, in the Beauville case,

$$
4 \chi(S)=4\left(1+p_{g}\right)=|G|\left(1-\mu_{1}\right)\left(1-\mu_{2}\right),
$$

where

$$
\mu_{i}:=\frac{1}{m_{1, i}}+\frac{1}{m_{2, i}}+\frac{1}{m_{3, i}}, \quad(i=1,2) .
$$

The most important property of surfaces isogenous to a product is their weak rigidity property.

Theorem 2.5. Cat03b, Theorem 3.3, Weak Rigidity Theorem] Let $S=\left(C_{1} \times C_{2}\right) / G$ be a surface isogenous to a higher product of curves. Then every surface with the same

- topological Euler number and

- fundamental group

is diffeomorphic to $S$. The corresponding moduli space $\mathcal{M}^{\text {top }}(S)=\mathcal{M}^{\text {diff }}(S)$ of surfaces (orientedly) homeomorphic (resp. diffeomorphic) to $S$ is either irreducible and connected or consists of two irreducible connected components exchanged by complex conjugation.

Thanks to the Weak Rigidity Theorem, we have that the moduli space of surfaces isogenous to a product of curves with fixed invariants - a finite group $G$ and a type $\left(\tau_{1}, \tau_{2}\right)$ in the unmixed case - consists of a finite number of irreducible connected components of $\mathcal{M}$. More precisely, let $S$ be a surface isogenous to a product of curves of unmixed type with group $G$ and a pair of disjoint systems of generators of type $\left(\tau_{1}, \tau_{2}\right)$. By $(9)$ we have $\chi(S)=\chi\left(G,\left(\tau_{1}, \tau_{2}\right)\right)$, and consequently, by (5) $K_{S}^{2}=K^{2}\left(G,\left(\tau_{1}, \tau_{2}\right)\right)=8 \chi(S)$, and $e(S)=e\left(G,\left(\tau_{1}, \tau_{2}\right)\right)=4 \chi(S)$. Moreover the fundamental group of $S$ fits in the following exact sequence (cf. Cat00]):

$$
1 \longrightarrow \pi_{1}\left(C_{1}\right) \times \pi_{2}\left(C_{2}\right) \longrightarrow \pi_{1}(S) \longrightarrow G \longrightarrow 1 .
$$

Let us fix a group $G$ and a type $\left(\tau_{1}, \tau_{2}\right)$ of an unmixed ramification structure, and denote by $\mathcal{M}_{\left(G,\left(\tau_{1}, \tau_{2}\right)\right)}$ the moduli space of isomorphism classes of surfaces isogenous to a product of curves of unmixed type admitting these data, then it is obviously a subset of the moduli space $\mathcal{M}_{K^{2}\left(G,\left(\tau_{1}, \tau_{2}\right)\right), \chi\left(G,\left(\tau_{1}, \tau_{2}\right)\right)}$. By the Weak Rigidity Theorem, the space $\mathcal{M}_{\left(G,\left(\tau_{1}, \tau_{2}\right)\right)}$ consists of a finite number of irreducible connected components.

A group theoretical method to count the number of these components is given in $\mathrm{BC} 02$, Theorem 1.3] in case of surfaces isogenous to a product of curves of unmixed type with $q=0$ and $G$ abelian. The following theorem is a natural generalization.

Theorem 2.6. [P11, Theorem 5.7] Let $S$ be a surface isogenous to a product of unmixed type. Then we attach to $S$ its finite group $G$ (up to isomorphism) and the equivalence class ramification structures $\left(\mathcal{V}_{1}, \mathcal{V}_{2}\right)$ of type $\left(\tau_{1}, \tau_{2}\right)$ of $G$, under the equivalence relation generated by:

(1) Hurwitz moves and $\operatorname{Inn}(G)$ on $\mathcal{V}_{1}$,

(2) Hurwitz moves and $\operatorname{Inn}(G)$ on $\mathcal{V}_{2}$,

(3) simultaneous conjugation of $\mathcal{V}_{1}$ and $\mathcal{V}_{2}$ by an element $\phi \in \operatorname{Aut}(G)$, i.e., we let $\left(\mathcal{V}_{1}, \mathcal{V}_{2}\right)$ be equivalent to $\left(\phi\left(\mathcal{V}_{1}\right), \phi\left(\mathcal{V}_{2}\right)\right)$.

Then two surfaces $S$ and $S^{\prime}$ are deformation equivalent if and only if the corresponding pairs of systems of generators are in the same equivalence class. 
If we fix a finite group $G$ and a pair of types $\left(\tau_{1}, \tau_{2}\right)$ of an unmixed ramification structure for $G$, counting the number of connected components of $\mathcal{M}_{\left(G,\left(\tau_{1}, \tau_{2}\right)\right)}$ is then equivalent to the group theoretical problem of counting the number of classes of pairs of systems of generators of $G$ of type $\left(\tau_{1}, \tau_{2}\right)$ under the equivalence relation defined in Theorem 2.6. This leads also to the following definition.

Definition 2.7. Denote by $h\left(G ; \tau_{1}, \tau_{2}\right)$ the number of Hurwitz components, namely the number of orbits of $\mathcal{U}\left(G ; \tau_{1}, \tau_{2}\right)$ under the action of the group prescribed in Theorem 2.6.

\section{Connected components of the Moduli Space of Surfaces of General Type}

We can simplify a lot the discussion of the previous section if we consider only regular surfaces, which will be assumed in the whole section. In this case, the Hurwitz moves are given only by the $\mathbf{t}_{\sigma_{i}}$ of Proposition 1.14, which corresponds to the ones described in Proposition 1.16. By Theorem 1.2 they are given by the braid group of the sphere on $r$ strands $\mathbf{B}_{r}$ acting on the generators of the orbifold fundamental group as in Figure 8.

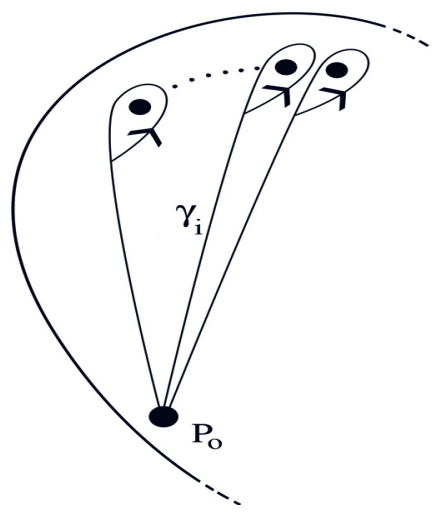

Figure 8.

In addition, we recall

Lemma 3.1. [V96, Lemma 9.4] The inner automorphism group, $\operatorname{Inn}(G)$, leaves each braid orbit invariant.

This lemma allows us to use the above Theorem 2.6 in the simplified version without the $\operatorname{Inn}(G)$ action on the system of generators $\mathcal{V}_{i}$. Since the two actions of $\mathbf{B}_{r}$ and $\operatorname{Aut}(G)$ commute, one gets a double action of $\mathbf{B}_{r} \times \operatorname{Aut}(G)$ on the set of $r$-systems of generators for $G$.

Therefore, for regular surfaces isogenous to a product we have that fixing a finite group $G$ and a pair of types $\left(\tau_{1}, \tau_{2}\right)=\left(m_{1,1}, \ldots, m_{1, r_{1}}, m_{2,1}, \ldots, m_{2, r_{2}}\right)$ of an unmixed ramification structure for $G$ counting the number of connected components of $\mathcal{M}_{\left(G,\left(\tau_{1}, \tau_{2}\right)\right)}$ is then equivalent to the group theoretical problem of counting the number of classes of pairs of systems of generators of $G$ of type $\left(\tau_{1}, \tau_{2}\right)$ under the equivalence relation given by the action of $\mathbf{B}_{r_{1}} \times \mathbf{B}_{r_{2}} \times \operatorname{Aut}(G)$. In this case the number of Hurwitz components $h\left(G ; \tau_{1}, \tau_{2}\right)$ is given by the number of orbits of $\mathcal{U}\left(G ; \tau_{1}, \tau_{2}\right)$ under the following actions:

if $\tau_{1} \neq \tau_{2}$ : the action of $\left(\mathbf{B}_{r_{1}} \times \mathbf{B}_{r_{2}}\right) \times \operatorname{Aut}(G)$, given by:

$$
\left(\left(\gamma_{1}, \gamma_{2}\right), \phi\right) \cdot\left(T_{1}, T_{2}\right):=\left(\phi\left(\gamma_{1}\left(T_{1}\right)\right), \phi\left(\gamma_{2}\left(T_{2}\right)\right)\right),
$$

where $\gamma_{1} \in \mathbf{B}_{r_{1}}, \gamma_{2} \in \mathbf{B}_{r_{2}}, \phi \in \operatorname{Aut}(G)$ and $\left(T_{1}, T_{2}\right) \in \mathcal{U}\left(G ; \tau_{1}, \tau_{2}\right)$.

if $\tau_{1}=\tau_{2}$ : the action of $\left(\mathbf{B}_{r} \prec \mathbb{Z} / 2 \mathbb{Z}\right) \times \operatorname{Aut}(G)$, where $\mathbb{Z} / 2 \mathbb{Z}$ acts on $\left(T_{1}, T_{2}\right)$ by exchanging the two factors.

In case of Beauville surfaces we define $h$ as above substituting $r_{1}$ and $r_{2}$ with 3 .

Proposition 3.2. Fix $r_{1}$ and $r_{2}$ in $\mathbb{N}$. Let $\left\{G_{n}\right\}_{n=1}^{\infty}$ be a family of finite groups, which admit an unmixed ramification structure of size $\left(r_{1}, r_{2}\right)$. Let $\tau_{n, 1}=\left(m_{n, 1,1}, \ldots, m_{n, 1, r_{1}}\right)$ and $\tau_{n, 2}=$ $\left(m_{n, 2,1}, \ldots, m_{n, 2, r_{2}}\right)$ be sequences of types $\left(\tau_{n, 1}, \tau_{n, 2}\right)$ of unmixed ramification structures for $G_{n}$, 
and $\left\{S_{n}\right\}_{n=1}^{\infty}$ be the family of surfaces isogenous to product with $q=0$ admitting the given data, then as $\left|G_{n}\right| \stackrel{n \rightarrow \infty}{\longrightarrow} \infty$ :

(1) $\chi\left(S_{n}\right)=\Theta\left(\left|G_{n}\right|\right)$.

(2) $h\left(G_{n} ; \tau_{n, 1}, \tau_{n, 2}\right)=O\left(\chi\left(S_{n}\right)^{r_{1}+r_{2}-2}\right)$.

Proof. (1) Note that, for $i=1,2$,

$$
\frac{1}{42} \leq-2+\sum_{j=1}^{r_{i}}\left(1-\frac{1}{m_{n, i, j}}\right) \leq r_{i}-2
$$

Indeed, for $r_{i}=3$, the minimal value for $\left(1-\mu_{i}\right)$ is $1 / 42$. For $r_{i}=4$, the minimal value for $\left(-2+\sum_{j=1}^{r_{i}}\left(1-\frac{1}{m_{n, i, j}}\right)\right)$ is $1 / 6$, and when $r_{i} \geq 5$, this value is at least $1 / 2$.

Now, by Equation (9),

$$
4 \chi\left(S_{n}\right)=\left|G_{n}\right| \cdot\left(-2+\sum_{j=1}^{r_{1}}\left(1-\frac{1}{m_{n, 1, j}}\right)\right) \cdot\left(-2+\sum_{j=1}^{r_{2}}\left(1-\frac{1}{m_{n, 2, j}}\right)\right),
$$

hence

$$
\frac{\left|G_{n}\right|}{4 \cdot 42^{2}} \leq \chi\left(S_{n}\right) \leq \frac{\left(r_{1}-2\right)\left(r_{2}-2\right)\left|G_{n}\right|}{4} .
$$

(2) For $i=1,2$, any $r_{i}$-system of generators $\mathcal{V}_{n, i}$ contains at most $r_{i}-1$ independent elements of $G_{n}$. Thus, the size of the set of all unordered pairs of type $\left(\tau_{n, 1}, \tau_{n, 2}\right)$ is bounded from above, by

$$
\left|\mathcal{U}\left(G_{n} ; \tau_{n, 1}, \tau_{n, 2}\right)\right| \leq\left|G_{n}\right|^{r_{1}+r_{2}-2},
$$

and so, the number of connected components is bounded from above by

$$
h\left(G_{n} ; \tau_{n, 1}, \tau_{n, 2}\right) \leq\left|G_{n}\right|^{r_{1}+r_{2}-2} .
$$

Now, the result follows from (1).

Therefore we cannot expect more than a polynomial growth in the number of connected components of surfaces of general type if we count only regular surfaces isogenous to a product. Together with Shelly Garion we investigated the asymptotic growth of the number of connected components of the moduli space of surfaces of general type by using the group theoretical methods described above for regular surfaces isogenous to a product. Our first results already appeared on the ArXiv in 2009 (see arXiv:0910.5402 v1). The following are some of the results contained in GP11.

Notation 3.3. Denote:

- $h(n)=\Omega(g(n))$, if $h(n) \geq c g(n)$ for some positive constant $c$, as $n \rightarrow \infty$.

- $h(n)=\Theta(g(n))$, if $c_{1} g(n) \leq h(n) \leq c_{2} g(n)$ for some positive constants $c_{1}, c_{2}$, as $n \rightarrow \infty$.

Theorem 3.4. Let $\tau_{1}=\left(m_{1,1}, \ldots, m_{1, r_{1}}\right)$ and $\tau_{2}=\left(m_{2,1}, \ldots, m_{2, r_{2}}\right)$ be two sequences of natural numbers such that $m_{k, i} \geq 2$ and $\sum_{i=1}^{r_{k}}\left(1-1 / m_{k, i}\right)>2$ for $k=1,2$. Let $h\left(\mathfrak{A}_{n}, \tau_{1}, \tau_{2}\right)$ be the number of connected components of the moduli space of surfaces isogenous to a product with $q=0$, with group the alternating group $\mathfrak{A}_{n}$, and with type $\left(\tau_{1}, \tau_{2}\right)$. Then

$$
\text { (a) } h\left(\mathfrak{A}_{n}, \tau_{1}, \tau_{2}\right)=\Omega\left(n^{r_{1}+r_{2}}\right),
$$

and moreover,

$$
\text { (b) } h\left(\mathfrak{A}_{n}, \tau_{1}, \tau_{2}\right)=\Omega\left((\log (\chi))^{r_{1}+r_{2}-\epsilon}\right) \text {. }
$$

where $0<\epsilon \in \mathbb{R}$. 
Theorem 3.5. Let $\tau_{1}=\left(m_{1,1}, \ldots, m_{1, r_{1}}\right)$ and $\tau_{2}=\left(m_{2,1}, \ldots, m_{2, r_{2}}\right)$ be two sequences of natural numbers such that $m_{k, i} \geq 2$, at least two of $\left(m_{k, 1}, \ldots, m_{k, r_{k}}\right)$ are even and $\sum_{i=1}^{r_{k}}\left(1-1 / m_{k, i}\right)>2$, for $k=1,2$. Let $h\left(\mathfrak{S}_{n}, \tau_{1}, \tau_{2}\right)$ be the number of connected components of the moduli space of surfaces isogenous to a product with $q=0$, with group the symmetric group $\mathfrak{S}_{n}$, and with type $\left(\tau_{1}, \tau_{2}\right)$. Then

$$
\text { (a) } h\left(\mathfrak{S}_{n}, \tau_{1}, \tau_{2}\right)=\Omega\left(n^{r_{1}+r_{2}}\right),
$$

and moreover,

$$
\text { (b) } h\left(\mathfrak{S}_{n}, \tau_{1}, \tau_{2}\right)=\Omega\left((\log (\chi))^{r_{1}+r_{2}-\epsilon}\right) \text {. }
$$

where $0<\epsilon \in \mathbb{R}$.

The proofs of part (a) of both Theorems are presented in [GP11, Section 3.2], and are based on results of Liebeck and Shalev [LS04]. The proofs of part (b) of both theorems appear in GP11, Section 2].

We can specialize the results above to Beauville surfaces. Recall that a triple $(r, s, t) \in \mathbb{N}^{3}$ is said to be hyperbolic if

$$
\frac{1}{r}+\frac{1}{s}+\frac{1}{t}<1 .
$$

Corollary 3.6. Let $\tau_{1}=\left(r_{1}, s_{1}, t_{1}\right)$ and $\tau_{2}=\left(r_{2}, s_{2}, t_{2}\right)$ be two hyperbolic types and let $h\left(\mathfrak{A}_{n}, \tau_{1}, \tau_{2}\right)$ be the number of Beauville surfaces with group $\mathfrak{A}_{n}$ and with types $\left(\tau_{1}, \tau_{2}\right)$. Then

$$
\text { (a) } h\left(\mathfrak{A}_{n}, \tau_{1}, \tau_{2}\right)=\Omega\left(n^{6}\right),
$$

and moreover,

$$
\text { (b) } h\left(\mathfrak{A}_{n}, \tau_{1}, \tau_{2}\right)=\Omega\left((\log (\chi))^{6-\epsilon}\right) \text {. }
$$

where $0<\epsilon \in \mathbb{R}$.

Corollary 3.7. Let $\tau_{1}=\left(r_{1}, s_{1}, t_{1}\right)$ and $\tau_{2}=\left(r_{2}, s_{2}, t_{2}\right)$ be two hyperbolic types, assume that at least two of $\left(r_{1}, s_{1}, t_{1}\right)$ are even and at least two of $\left(r_{2}, s_{2}, t_{2}\right)$ are even, and let $h\left(\mathfrak{S}_{n}, \tau_{1}, \tau_{2}\right)$ be the number of Beauville surfaces with group $\mathfrak{S}_{n}$ and with types $\left(\tau_{1}, \tau_{2}\right)$. Then

$$
\text { (a) } h\left(\mathfrak{S}_{n}, \tau_{1}, \tau_{2}\right)=\Omega\left(n^{6}\right),
$$

and moreover,

$$
\text { (b) } h\left(\mathfrak{S}_{n}, \tau_{1}, \tau_{2}\right)=\Omega\left((\log (\chi))^{6-\epsilon}\right) \text {. }
$$

where $0<\epsilon \in \mathbb{R}$.

The situation is more interesting for abelian groups. We have the following results which assure the existence of ramification structure for abelian group.

Theorem 3.8. Let $G$ be an abelian group, given as

$$
G \cong \mathbb{Z} / n_{1} \mathbb{Z} \times \cdots \times \mathbb{Z} / n_{t} \mathbb{Z},
$$

where $n_{1}|\cdots| n_{t}$. For a prime $p$, denote by $l_{i}(p)$ the largest power of $p$ which divides $n_{i}$ (for $1 \leq i \leq t)$

Let $r_{1}, r_{2} \geq 3$, then $G$ admits an unmixed ramification structure of size $\left(r_{1}, r_{2}\right)$ if and only if the following conditions hold:

- $r_{1}, r_{2} \geq t+1$

- $n_{t}=n_{t-1}$;

- If $l_{t-1}(3)>l_{t-2}(3)$ then $r_{1}, r_{2} \geq 4$;

- $l_{t-1}(2)=l_{t-2}(2)$

- If $l_{t-2}(2)>l_{t-3}(2)$ then $r_{1}, r_{2} \geq 5$ and $r_{1}, r_{2}$ are not both odd.

This theorem is proved in [GP11, Section 3.4]. Once we established the existence of ramification structures, we can put the surfaces in sequences and compute the number of connected components of the moduli space. More precisely, the following holds. 
Theorem 3.9. Let $\left\{S_{p}\right\}$ be the family of surfaces isogenous to a product with $q=0$ with group $G_{p}:=(\mathbb{Z} / p \mathbb{Z})^{r}$ admitting ramification structure of type $\tau_{p}=(p, \ldots, p)(p$ appears $(r+1)$-times) where $p$ is prime. If we denote by $h\left(G_{p} ; \tau_{p}, \tau_{p}\right)$ the number of connected components of the moduli space of isomorphism classes of surfaces isogenous to a product with $q=0$ admitting these data, then

$$
h\left(G_{p} ; \tau_{p}, \tau_{p}\right)=\Theta\left(\chi^{r}\left(S_{p}\right)\right) .
$$

Therefore, there exist families of surfaces such that the degree of the polynomial $h$ in $\chi$ (and so in $K^{2}$ ) can be arbitrarily large. The proof of this theorem appears in GP11, Section $2]$. Notice that not only the number of connected components increases, but also their sizes. Indeed, we see that $2 r-6$ is the dimension of these connected components.

Again we can specialize the results for Beauville surfaces.

Corollary 3.10. Let $\left\{S_{p}\right\}$ be the family of Beauville surfaces $G_{p}:=(\mathbb{Z} / p \mathbb{Z})^{2}$ admitting ramification structure of type $\tau_{p}=(p, p, p)$ where $p \geq 5$ is prime. If we denote by $h\left(G_{p} ; \tau_{p}, \tau_{p}\right)$ the number of Beauville surfaces admitting these data. Then

$$
h\left(G_{p} ; \tau_{p}, \tau_{p}\right)=\Theta\left(\chi^{2}\left(S_{p}\right)\right) .
$$

Proof. Let $\left(x_{1}, x_{2} ; y_{1}, y_{2}\right)$ be an unmixed Beauville structure for $G$. Since $x_{1}, x_{2}$ are generators of $G$, they are a basis, and without loss of generality $x_{1}, x_{2}$ are the standard basis $x_{1}=(1,0)$, $x_{2}=(0,1)$. Now, let $y_{1}=(a, b), y_{2}=(c, d)$, then the condition (7) means that any pair of the six vectors yield a basis of $G$, implying that $a, b, c, d$ must satisfy the following conditions

$$
a-b, a+c, c-d, b+d, a+c-b-d, a d-b c \in U
$$

Moreover, the number $N_{p}$ of quadruples $(a, b, c, d)$ satisfy $(11)$ is $N_{p}=(p-1)(p-2)(p-$ $3)(p-4)$. The pairs $((1,0),(0,1) ;(a, b),(c, d))$, where $a, b, c, d$ satisfy (11), are exactly the representatives for the $\operatorname{Aut}(G)$-orbits in the set $\mathcal{U}(G ; \tau, \tau)$.

Now, one should consider the action of $\mathbf{B}_{3} \times \mathbf{B}_{3}$ on $\mathcal{U}(G ; \tau, \tau)$, which is equivalent to the action of $\mathfrak{S}_{3} \times \mathfrak{S}_{3}$, since $G$ is abelian. The action of $\mathfrak{S}_{3}$ on the second component is obvious (there are 6 permutations), and the action of $\mathfrak{S}_{3}$ on the first component can be translated to an equivalent $\operatorname{Aut}(G)$-action, given by multiplication in one of the six matrices:

$$
\left(\begin{array}{ll}
1 & 0 \\
0 & 1
\end{array}\right),\left(\begin{array}{ll}
0 & 1 \\
1 & 0
\end{array}\right),\left(\begin{array}{ll}
-1 & 0 \\
-1 & 1
\end{array}\right),\left(\begin{array}{ll}
1 & -1 \\
0 & -1
\end{array}\right),\left(\begin{array}{ll}
-1 & 1 \\
-1 & 0
\end{array}\right),\left(\begin{array}{ll}
0 & -1 \\
1 & -1
\end{array}\right),
$$

yielding an equivalent representative.

Therefore, the action of $\mathfrak{S}_{3}$ on the second component yields orbits of length 6 , and the action of $\mathfrak{S}_{3}$ on the first component connects them together, and gives orbits of sizes from 6 to 36 . Moreover since one can exchange the vector $\left(x_{1}, x_{2}\right)$ with the vector $\left(y_{1}, y_{2}\right)$ we get

$$
N_{p} / 72 \leq h \leq N_{p} / 6 .
$$

By Proposition 3.2, we have as $p \rightarrow \infty$ :

$$
\chi\left(S_{p}\right)=\Theta\left(p^{2}\right),
$$

while by the above computation we have

$$
h\left(G_{p} ; \tau_{p}, \tau_{p}\right)=\Theta\left(p^{4}\right) .
$$

Therefore

$$
h\left(G_{p} ; \tau_{p}, \tau_{p}\right)=\Theta\left(\chi^{2}\left(S_{p}\right)\right)
$$

Corollary 3.11. Let $n$ be an integer such that $(n, 6)=1$. The number $h=h(G ; \tau, \tau)$, where $\tau=(n, n, n)$, of Hurwitz components for $G=(\mathbb{Z} / n \mathbb{Z})^{2}$, where $n=p_{1}^{k_{1}} \cdot \ldots \cdot p_{t}^{k_{t}}$, satisfies

$$
N_{n} / 72 \leq h \leq N_{n} / 6,
$$

where $N_{n}=\prod_{i=1}^{t} p_{i}^{4 k_{i}-4}\left(p_{i}-1\right)\left(p_{i}-2\right)\left(p_{i}-3\right)\left(p_{i}-4\right)$. 
Remark 3.12. Notice that if $n$ is divisible by the first $l$ primes $p_{i} \geq 5$ then since:

$$
\lim _{l \rightarrow \infty} \prod_{i}\left(1-\frac{1}{p_{i}}\right)=0
$$

we have $N_{n} / n^{4} \rightarrow 0$ as $l \rightarrow \infty$.

In GJT11 the authors give an explicit formula for the number of isomorphism classes of Beauville surfaces $\Theta(n)$, which we now explain. We shall keep the notation of GJT11. Define the following functions for $n, e \in \mathbb{N}$ and $p$ prime.

$$
\begin{aligned}
& \Theta_{1}(n):=n^{4} \prod_{p \mid n}\left(1-\frac{1}{p}\right)\left(1-\frac{2}{p}\right)\left(1-\frac{3}{p}\right)\left(1-\frac{4}{p}\right) ; \\
& \Theta_{2}\left(p^{e}\right):=\left\{\begin{array}{llll}
p^{2 e}\left(1-\frac{1}{p}\right)\left(1-\frac{2}{p}\right) & \text { if } & p \equiv 1 \quad \bmod 4 \\
p^{2 e}\left(1-\frac{1}{p}\right)\left(1-\frac{4}{p}\right) & \text { if } & p \equiv 3 \quad \bmod 4
\end{array}\right. \\
& \Theta_{3}\left(p^{e}\right):=p^{2 e}\left(1-\frac{3}{p}\right)\left(1-\frac{5}{p}\right) ; \\
& \Theta_{4}\left(p^{e}\right):=\left\{\begin{array}{lll}
0 & \text { if } & p \equiv-1 \quad \bmod 3 \\
2 & \text { if } & p \equiv 1
\end{array}\right.
\end{aligned}
$$

Theorem 3.13. GJT11, Theorem 2] Let $n=p_{1}^{e_{1}} \cdot \ldots \cdot p_{t}^{e_{t}}$ be an integer such that $(n, 6)=1$. Then the number of isomorphism classes of Beauville surfaces with group $G=(\mathbb{Z} / n \mathbb{Z})^{2}$ is

$$
\Theta(n):=\frac{1}{72}\left(\Theta_{1}(n)+4 \prod_{i=1}^{t} \Theta_{2}\left(p_{i}^{e_{i}}\right)+6 \prod_{i=1}^{t} \Theta_{3}\left(p_{i}^{e_{i}}\right)+12 \prod_{i=1}^{t} \Theta_{4}\left(p_{i}^{e_{i}}\right)\right) .
$$

The case of irregular surfaces has not been so intensively investigated. Indeed, counting the number of connected components of the moduli space of surfaces isogenous to a product with fixed data can be more complicated. We have to consider Theorem 2.6 together with the Hurwitz moves described in Proposition 1.14. Indeed, this procedure was applied in two specific cases: For surfaces isogenous to a product with $p_{g}=q=1$ or 2 in [Po08] and in [P11]. In both cases it was used a GAP4 script which can be found in [P10]. It would be interesting to consider all the surfaces isogenous to a product with fixed invariants $K^{2}$ and $\chi$ and count the number of connected components of the moduli space of surfaces of general type they give. Indeed for $K^{2}=8$ and $\chi=1$ we can count already 94 connected componens. Respectively: 1 component with $p_{g}=q=4,1$ with $p_{g}=q=3,27$ with $p_{g}=q=2,52$ with $p_{g}=q=1$ and 13 with $p_{g}=q=0$.

In this paper we consider only unmixed surfaces isogenous to a product, nevertheless similar methods could be applied also for the mixed case. Indeed, there are already works in these direction, see [FP13].

\section{REFERENCES}

[BC02] I. Bauer, F. Catanese, Some new surfaces with $p_{g}=q=0$. Proceeding of the Fano Conference. Torino (2002), 123-142.

[BCG05] I. Bauer, F. Catanese, F. Grunewald, Beauville surfaces without real structures. In: Geometric methods in algebra and number theory, Progr. Math., 235, Birkhäuser Boston, (2005), 1-42.

[BCG06] I. Bauer, F. Catanese, F. Grunewald, Chebycheff and Belyi polynomials, dessins d'enfants, Beauville surfaces and group theory. Mediterr. J. Math. 3, (2006), 121-146.

[BCG08] I. Bauer, F. Catanese, F. Grunewald, The classification of surfaces with $p_{g}=q=0$ isogenous to a product. Pure Appl. Math. Q., 4, (2008), 547-586.

[Bir69] J.S. Birman, Mapping Class Groups and Their Relationship to Braid Groups. Comm. on Pure and Applied Math XXII, (1969), 213-238.

[Bir74] J.S. Birman, Braids, links, and mapping class groups. Annals of Mathematics Studies, No. 82, Princeton University Press (1974).

[Cat92] F. Catanese, Chow varieties, Hilbert schemes and moduli spaces of surfaces of general type. J. Algebraic Geom. 1 (1992), 561-595. 
[Cat00] F. Catanese, Fibred surfaces, varieties isogenous to a product and related moduli spaces. Amer. J. Math. 122, (2000), 1-44.

[Cat03a] F. Catanese, Differentiable and deformation type of algebraic surfaces, real and symplectic structures. Symplectic 4-manifolds and algebraic surfaces. Lectures given at the C.I.M.E. summer school, Cetraro, Italy, September 2-10 2003. Lecture Notes in Mathematics 1938 Springer, Berlin (2008), 55-167.

[Cat03b] F, Catanese, Moduli spaces of surfaces and real structures. Ann. of Math. 158, (2003), 577-592.

[Cat05] F. Catanese, Trecce, mapping class groups, fibrazioni di Lefschetz e applicazioni al diffeomorfismo di superfici algebriche. Luigi Cremona (1830-1903) Incontr. Studio 36. Milano (2005), 207-235.

[CLP11] F. Catanese, M. Lönne, F. Perroni Irreducibility of the space of dihedral cover of the projective line of a given numerical type, arXiv:1206.5498

[D38] M. Dehn, Die Gruppe der Abbildungsklassen. (Das arithmetische Feld auf Flächen.) Acta Math. 69, (1938), $135-206$

[FP13] D. Frapporti, R. Pignatelli Mixed quasi-ṫale quotients with arbitrary singularities, arXiv:1302.3717

[GP09] S. Garion, M. Penegini New Beauville surfaces and finite simple groups. Preprint (2009) to appear in Manuscripta Math., DOI: 10.1007/s00229-013-0607-0

[GP11] S. Garion, M. Penegini Beauville surfaces, moduli spaces and finite groups preprint (2011) to appear in Comm. in Algebra.

[GJT11] G. Gonzales-Diez, G. Jones, D. Torres-Teigell Beauville surfaces with abelian Beauville group. Preprint, ArXiv: $1102.4552 \mathrm{v} 3$.

[LS04] M.W. Liebeck, A. Shalev, Fuchsian groups, coverings of Riemann surfaces, subgroup growth, random quotients and random walks. J. Algebra 276 (2004) 552-601.

[LS05] M.W. Liebeck, A. Shalev, Fuchsian groups, finite simple groups and representation varieties. Invent. Math. 159 (2005), no. 2, 317-367.

[Macl74] C. Maclachlan, Modular groups and fiber spaces over Teichmüller spaces. Discontin. Groups Riemann Surf., Proc. 1973 Conf. Univ. Maryland, (1974), 297-314.

[M97] M. Manetti, Iterated double covers and connected components of moduli spaces. Topology 36, (1997), $745-764$.

[P11] M. Penegini, The classification of isotrivially fibred surfaces with $p_{g}=q=2$. With an Appendix of S. Rollenske Collect. Math. 62, (2011), 239-274.

[P10] M. Penegini, The classification of isotrivially fibred surfaces with $p_{g}=q=2$, and topics on Beauville surfaces. Ph.D Thesis Universität Bayretuh, (2010).

[Po08] F. Polizzi, On surfaces of general type with $p_{g}=q=1$ isogenous to a product of curves. Comm. Algebra 36, (2008), 2023-2053.

[V96] H. Völklein, Groups as Galois groups - an introduction. Cambridge Studies in Advanced Mathematics, 53. Cambridge University Press, Cambridge, 1996.

Matteo Penegini, Dipartimento di Matematica "F. Enriques", Via Saldini 50, I-20133 Milano, ITALY

E-mail address: matteo.penegini@unimi.it 